\title{
PHASE-TYPE APPROXIMATIONS TO FINITE-TIME RUIN \\ PROBABILITIES IN THE SPARRE-ANDERSEN AND \\ STATIONARY RENEWAL RISK MODELS
}

BY

\author{
D.A. Stanford, F. Avram, A.L. Badescu, L. Breuer, \\ A. DA Silva Soares And G. Latouche
}

\begin{abstract}
The present paper extends the "Erlangization" idea introduced by Asmussen, Avram, and Usabel (2002) to the Sparre-Andersen and stationary renewal risk models. Erlangization yields an asymptotically-exact method for calculating finite time ruin probabilities with phase-type claim amounts. The method is based on finding the probability of ruin prior to a phase-type random horizon, independent of the risk process. When the horizon follows an Erlang- $l$ distribution, the method provides a sequence of approximations that converges to the true finite-time ruin probability as $l$ increases. Furthermore, the random horizon is easier to work with, so that very accurate probabilities of ruin are obtained with comparatively little computational effort. An additional section determines the phase-type form of the deficit at ruin in both models. Our work exploits the relationship to fluid queues to provide effective computational algorithms for the determination of these quantities, as demonstrated by the numerical examples.
\end{abstract}

\section{KEYWORDS}

Sparre-Andersen model, ladder height, maximal aggregate loss, deficit at ruin, phase-type distribution.

\section{INTRODUCTION}

The present paper is concerned with the determination of the probability of ruin in finite time in the Sparre-Andersen risk model and the stationary renewal risk model (also known as the stationary Sparre-Andersen model).

Traditionally, the exact determination of finite time ruin probabilities, in both the classical and the Sparre-Andersen risk models, has required the solution of rather complicated integro-differential equations. Explicit formulae for the probability of ruin exist for a limited number of cases, such as the classical model with exponential claim sizes. Even then, the form of the solution entails the evaluation of Bessel functions (see, for instance, Drekic \& Willmot (2003)). 
than the regular values for $l=7$ when $T=10$, which is atypical. Furthermore, the extrapolated value for $l=6$ when $T=50$ actually exceeds the ultimate ruin probability very slightly. Nonetheless, we would submit that the accuracy of the results for $l=7$ is already quite sufficient for any decision making purpose.

The numbers for this second example were run completely using the more time-consuming Mathematica code, yet still each value for $l=7$ ran in about 30 seconds.

\section{ACKNOWLEDGEMENTS}

The work of Dr. Stanford has been supported by a "Research in Brussels" grant from the Gouvernement de la Région de Bruxelles-Capitale, as well as his NSERC Discovery grant, which has also supported the doctoral work of Dr. Badescu. The work of Dr. Breuer has been supported by the "Prix Ouverture International" of the Université Libre de Bruxelles. We also wish to thank the referees for their comments, which have improved the paper.

Dr. Stanford also wishes to thank the Department of Statistics at the London School of Economics for technical support during the writing of this paper.

\section{REFERENCES}

Asmussen, S. (2000) Ruin Probabilities. World Scientific, Singapore.

Asmussen, S., Avram, F., and Usabel, M. (2002) Erlangian approximations for finite-horizon ruin probabilities. ASTIN Bulletin, 32(2), 267-281.

Asmussen, S. \& HøjgaARD, B. (1999) Approximations for finite-horizon ruin probabilities in the renewal model. Scand. Act. J., 1999, No. 2, 106-119.

Asmussen, S. and Rolski, T. (1991) Computational methods in risk theory: a matrix-algorithmic approach. Insurance: Mathematics and Economics, 10, 259-274.

Avram, F. and Usabel, M. (2003) Finite time ruin probabilities with one Laplace inversion. Insurance: Mathematics and Economics, 32, 371-377.

Badescu, A., Breuer, L., da Silva Soares, A., Latouche, G., Remiche, M.-A. and StanFORD, D.A. (2004) Risk Processes Analyzed as Fluid Queues. Scand. Act. J., posted on "prEview" site September 9, 2004. 16 pages.

Da Silva Soares, A. and Latouche, G. (2002) Further results on the similarity between fluid queues and QBDs. In G. Latouche and P. Taylor, editors, Proceedings of the 4th International Conference on Matrix-Analytic Methods, pages 89-106. World Scientific, River Edge, NJ, 2002.

Dickson, D.C.M. and WATERS, H.R. (1991) Recursive calculation of survival probabilities. ASTIN Bulletin, 22, 199-221.

Drekic, S. and Willmot, G.E. (2003) On the density and moments of the time of ruin with exponential claims. ASTIN Bulletin, 33, 11-21.

Drekic, S., Dickson, D.C.M., Stanford, D.A., and Willmot, G.E. (2004) On the distribution of the deficit at ruin when claims are phase-type. Scand. Act. J., 2004, 105-121.

Latouche, G. and Ramaswami, V. (1999) Introduction to Matrix Analytic Methods in Stochastic Modeling. ASA SIAM, Philadelphia.

Neuts, M.F. (1981) Matrix-Geometric Solutions in Stochastic Models. Johns Hopkins University Press, Baltimore.

Rolski, T., Schmidli, H., Schmidt, V., and Teugels, J. (1999) Stochastic Processes for Insurance and Finance. John Wiley, Chichester.

STANFORD, D.A. and StroińSKi, K.J. (1994) Recursive method for computing finite-time ruin probabilities for phase-distributed claim sizes. ASTIN Bulletin, 24, 235-254. 
StANFord, D.A., Stroiński, K.J., and LeE, K. (2000) Ruin probabilities based at claim instants for some non-Poisson claim processes. Insurance: Mathematics and Economics, 26, 251-267.

Thorin, O. and Wikstad, N. (1973) Numerical Evaluation of Ruin Probabilities for a finite Period. ASTIN Bulletin, 7, 137-153.

Thorin, O. and WiKsTAD, N. (1977) Calculation of ruin probabilities when the claim distribution is lognormal. ASTIN Bulletin, 9, 231-246.

WIKSTAD, N. (1971). Exemplification of ruin probabilities. ASTIN Bulletin, 6, 147-152.

Willmot, G.E., Dickson, D.C.M., Drekic, S., and Stanford, D.A. (2004) The deficit at ruin in the stationary renewal risk model. Scand. Act. J., 2004, 241-255.

David Stanford \& ANDrei Badescu ${ }^{\dagger}$

Dept. of Statistical \& Actuarial Sciences, WSC 262,

University of Western Ontario, London, Ontario N6A 5B7, Canada

E-mail:stanford@stats.uwo.ca, abadescu@math.uwaterloo.ca

$\dagger$ Presently in Dept. of Statistics \& Actuarial Science, U. Waterloo

Ana da Silva Soares \& Guy Latouche

Département d'Informatique, Université Libre de Bruxelles

Campus Plaine CP 212, B-1050 Bruxelles, Belgium

E-mail: andasilv@ulb.ac.be,latouche@ulb.ac.be

FLORIN AVRAM

Département de Mathématiques, Université de Pau

Bat. IPRA, Avenue de l'université, 64000 Pau, France

E-mail: Florin.Avram@univ-pau.fr

LOTHAR BREUER

Department IV - Computer Science, University of Trier

D-54286 Trier, Germany

E-mail:breuer@info04.uni-trier.de 